\title{
Correction to: Combination Therapy with Pirfenidone plus Prednisolone Ameliorates Paraquat-Induced Pulmonary Fibrosis
}

\author{
Rokhsana Rasooli, ${ }^{1}$ Fatemeh Pourgholamhosein, ${ }^{2}$ Younes Kamali, ${ }^{3}$ Fatemeh Nabipour, ${ }^{4}$ and \\ Ali Mandegary ${ }^{5,6}$
}

Correction to: Inflammation

https://doi.org/10.1007/s10753-017-0671-9

Unfortunately, the original publication of this article contained mistakes, and the authors would like to correct them. The corrected details are given below:

In the first column of Table 1, "Type 2 pneumocyte", should read as "Type 2 pneumocyte proliferation".

In Figure 3, the spelling of "Chenges" on the y-axis was incorrect and it should be spelled as "Changes".

The authors apologize for potential issues raised by these mistakes.

The online version of the original article can be found at https://doi.org/ 10.1007/s10753-017-0671-9

${ }^{1}$ Department of Pharmacology, School of Veterinary Medicine, Shiraz University, Shiraz, Iran

${ }^{2}$ Pharmaceutics Research Center, Institute of Neuropharmacology, Kerman University of Medical Sciences, Kerman, Iran

${ }^{3}$ Department of Anatomic Sciences, Anatomy and Embryology Division, School of Veterinary Medicine, Shiraz University, Shiraz, Iran

${ }^{4}$ Department of Pathology, School of Medicine, Kerman University of Medical Sciences, Kerman, Iran

${ }^{5}$ Neuroscience Research Center, Institute of Neuropharmacology, and Department of Pharmacology \& Toxicology, School of Pharmacy, Kerman University of Medical Sciences, Kerman, Iran

${ }^{6}$ To whom correspondence should be addressed at Neuroscience Research Center, Institute of Neuropharmacology, and Department of Pharmacology \& Toxicology, School of Pharmacy, Kerman University of Medical Sciences, Kerman, Iran. E-mail: alimandegary@kmu.ac.ir 